\title{
Genetic Interactions Among Ghd7, Ghd8, OsPRR37 and Hd1 Contribute to Large Variation in Heading Date in Rice
}

\author{
Bo Zhang ${ }^{1}$, Haiyang Liu', Feixiang Qi $i^{1}$, Zhanyi Zhang ${ }^{1}$, Qiuping $\mathrm{Li}^{1}$, Zhongmin $\mathrm{Han}^{1}$ and Yongzhong Xing ${ }^{1,2^{*}}$ (D)
}

\begin{abstract}
Background: Heading date is crucial for rice reproduction and geographic expansion. Many heading date genes are sensitive to photoperiod and jointly regulate flowering time in rice. However, it is not clear how these genes coordinate rice heading.

Results: Here, we performed a genetic interaction analysis among four major rice heading date genes Ghd7, Ghd8, OsPRR37/Ghd7.1 (hereafter PRR37) and Hd1 in the near-isogenic background under both natural long-day (NLD) and natural short-day (NSD) conditions. The 4-gene segregating population exhibited a large heading date variation with more than 95 days under NLD and 42 days under NSD conditions. Tetragenic, trigenic and digenic interactions among these four genes were observed under both conditions but more significant under NLD conditions. In the functional Hd1 backgrounds, the strongest digenic interaction was Ghd7 by Ghd8 under NLD but was Ghd7 by PRR37 under NSD conditions. Interestingly, PRR37 acted as a flowering suppressor under NLD conditions, while it functioned alternatively as an activator or a suppressor under NSD conditions depending on the status of the other three genes. Based on the performances of 16 homozygous four-gene combinations, a positive correlation between heading date and spikelets per panicle (SPP) was found under NSD conditions, but changed to a negative correlation when heading date was over 90 days under NLD conditions.
\end{abstract}

Conclusions: These results demonstrate the importance of genetic interactions in the rice flowering regulatory network and will help breeders to select favorable combinations to maximize rice yield potential for different ecological areas.

Keywords: Rice, Heading date, Genetic interaction, Alternative function, Genotype combination, Correlation, Spikelets per panicle

\section{Background}

Heading date, a crucial trait for rice expansion to high latitudes, is determined by both genetic factors and environmental cues (Andres and Coupland 2012). Cultivars with an appropriate heading date will be conductive to high grain yield by fully utilizing the light and temperature resources in their growing regions (Zhang et al. 2015a).

In the last two decades, dozens of quantitative trait loci (QTLs) for rice heading date have been cloned by

\footnotetext{
*Correspondence: yzxing@mail.hzau.edu.cn

${ }^{1}$ National Key Laboratory of Crop Genetic Improvement and National Center of Plant Gene Research (Wuhan), Huazhong Agricultural University, Wuhan 430070, China

${ }^{2}$ Hubei collaborative Innovation Center for Grain Industry, Yangtze University, Jingzhou 434100, China
}

using biparental populations, germplasm resources and mutants with forward- or reverse-genetics approaches (Yamamoto et al. 2012; Hori et al. 2016; Yano et al. 2016). Among these genes, several major QTLs, especially those cloned from natural variations, have pleiotropic effects on heading date, plant height and grain yield, which have been widely subjected to artificial selection in the process of rice genetic improvement. For example, Heading date1 (Hd1), the homolog of Arabidopsis CONSTANS $(\mathrm{CO})$, encodes a zinc finger CCT $(\mathrm{CO}, \mathrm{CO}$ LIKE and TIMING OF CAB1) domain and acts as a major flowering activator in rice (Yano et al. 2000; Zhang et al. 2017). Hd1 delays heading date in some varieties under long-day (LD) conditions by interacting with other flowering genes such as $G h d 7$, resulting in a taller plant and more grain yield (Nemoto et al. 2016; Zhang et al. 2017). Ghd7 is 
a rice-specific gene encoding a CCT domain protein and is important for heading date, grain yield, rice adaptation and drought resistance (Xue et al. 2008; Weng et al. 2014). Another major QTL, Ghd8 (allelic to $H d 5$ and DTH8), encodes a HAP3 subunit of heterotrimeric heme activator protein (HAP) and simultaneously controls heading date, plant height and grain number (Wei et al. 2010; Yan et al. 2011; Fujino et al. 2013). OsPRR37, allelic to Ghd7.1, $D T H 7$ and $H d 2$ and encoding a PSEUDO-RESPONSE REGULATOR 7-like protein harboring the CCT domain, greatly represses heading and increases grain yield under LD conditions (Koo et al. 2013; Liu et al. 2013; Gao et al. 2014). Natural variations in OsPRR37/Ghd7.1 also contribute to rice cultivation at a wide range of latitudes (Koo et al. 2013; Yan et al. 2013). It was initially demonstrated that these genes are in separate branches in the flowering regulatory network and have partially unrelated effects on transcription level (Brambilla and Fornara 2013; Song et al. 2015).

Photoperiod sensitivity largely determines heading date in rice. There are two independent genetic pathways involved in photoperiod sensitivity. One is the OsGI-Hd1-Hd3a pathway, which is conserved with the GI-CO-FT pathway in Arabidopsis (Shrestha et al. 2014). $H d 1$ is upregulated by $O s G I$ and activates the expression of $\mathrm{Hd} 3 \mathrm{a}$ to promote rice heading under both short-day (SD) and LD conditions (Hayama et al. 2003; Zhang et al. 2017). Another is the Ehd1-Hd3a pathway, a unique pathway in rice regulated by many genes (Doi et al. 2004; Tsuji et al. 2011). Among these genes, Ehd2, Ehd3, Ehd4 and OsMADS51 always promote rice heading by directly or indirectly upregulating the expression of Ehd1 under both SD and LD conditions (Kim et al. 2007; Matsubara et al. 2008; Matsubara et al. 2011; Gao et al. 2013). In contrast, other genes including Ghd7, Ghd8, OsPRR37, Hd16, OsCOL4 and OsCOL10 repress the expression of Ehd1, resulting in late flowering under LD conditions (Xue et al. 2008; Lee et al. 2010; Yan et al. 2011; Hori et al. 2013; Yan et al. 2013; Tan et al. 2016). The recent finding that the Ghd7-Hd1 complex represses $E h d 1$ by binding to a cis-regulatory region in the Ehd1 5'-UTR suggested that $H d 1$ was integrated into the rice-specific genetic pathway (Nemoto et al. 2016).

Our previous studies indicated that Ghd7 and Ghd8 in the ZS97 background greatly delayed heading date (nonheading) under NLD conditions because of the presence of $H d 1$, indicating a strong genetic interaction among Ghd7, Ghd8 and Hd1 (Zhang et al. 2015a). PRR37 shared the conserved CCT domain with Hd1 and Ghd7, and formed a heterotrimer with Ghd8 and NF-YCs similar to Hd1 (Zhang et al. 2015b; Goretti et al. 2017). Thus, we hypothesized that PRR37 is involved in genetic interactions with the three other genes. To test this hypothesis, we further conducted genetic interaction analysis among Ghd7, Ghd8, PRR37 and $H d 1$ in the ZS97 background under NLD and NSD conditions in this study. Tetragenic, trigenic and digenic interactions among these four genes were observed under both conditions. PRR37 always acts as a flowering suppressor under NLD conditions but exhibits an alternative function (either suppression or activation) in heading date under NSD conditions.

\section{Materials and methods}

Construction of NILs and segregating populations

We previously developed a near-isogenic line (NIL1) pyramiding functional Ghd $7^{M H 63}$ and Ghd8 $8^{9311}$ in the ZS97 background (Zhang et al. 2015a). Another nearisogenic line (NIL2) in the ZS97 background, which harbored functional $P R R 37^{T Q}$ and nonfunctional $h d 1^{T Q}$ derived from Teqing (TQ), was crossed with NIL1. Therefore, NIL- $\mathrm{F}_{1}$ plants carried heterozygous Ghd7, Ghd8, PRR37 and Hd1 (Additional file 1: Figure S1a; Table S1). The NIL-F $\mathrm{F}_{2}$ population was developed by selfcrossing a NIL- $\mathrm{F}_{1}$ plant that was genotyped by using the RICE6K SNP array (Yu et al. 2014) (Additional file 1: Figure S1b). To avoid genetic background noise, a NIL$\mathrm{F}_{2}$ individual harboring heterozygous alleles at all four of these genes was used to produce a NIL- $\mathrm{F}_{3}$ population by self-pollination. All individuals of the NIL- $\mathrm{F}_{2}$ and NIL- $\mathrm{F}_{3}$ populations were genotyped at these four gene loci. According to the genotypes of the NIL- $\mathrm{F}_{3}$ population, 8 NIL-F $\mathrm{F}_{3}$ plants, each carrying heterozygous PRR37 but with different homozygous combinations of the other three genes, were used to generate $8 \mathrm{NIL}^{-} \mathrm{F}_{4}$ populations for estimating the genetic effects of PRR37. Sixteen NIL$\mathrm{F}_{3}$ plants with different homozygous four-gene combinations were selected to generate 16 four-gene homozygous lines for evaluating yield performance.

\section{Field experiments and growth conditions}

Rice seeds were sown in a seedling bed in the middle of May at the experimental station of Huazhong Agricultural University, Wuhan, China $\left(30.5^{\circ} \mathrm{N}\right)$. The 25 -day-old seedlings were transplanted into the field with a distance of $16.5 \mathrm{~cm}$ between plants within a row and $26.5 \mathrm{~cm}$ between rows. The plants were subsequently grown in the field under NLD conditions (a day length of more than $13.5 \mathrm{~h}$ ) until the beginning of August (Additional file 1: Table S2). For the field experiments under NSD conditions, the plant materials were sown in Lingshui, Hainan $\left(18.5^{\circ} \mathrm{N}\right)$, at the beginning of December and were transplanted into the field after 1 month, at the same planting density as that used in Wuhan, and grown under an average day length of less than $12.5 \mathrm{~h}$ from December to April (Additional file 1: Table S2).

The NIL- $\mathrm{F}_{2}$ population consisting of 680 individuals was grown in Wuhan in 2016. Excluding the marginal 
plants and abnormally growing individuals, 509 individuals were used for analysis of genetic interactions among Ghd7, Ghd8, PRR37 and Hd1 under NLD conditions. A total of 900 NIL- $F_{3}$ plants derived from an $F_{2}$ individual segregating for these four genes were grown in Lingshui from Dec 2016 to Apr 2017, and a total of 679 non-marginal individuals were used for analysis of genetic interactions among these four genes under NSD conditions. Eight NIL-F 4 populations were grown in Wuhan ( 60 plants per population) in summer 2017 (from May to October) and in Lingshui ( 40 plants per population) in winter (from Dec 2017 to Apr 2018). Meanwhile, 16 four-gene homozygous lines were also grown in Wuhan and Lingshui in summer and winter of 2017, respectively. Three additional PRR37segregating population ( $\sim 80$ plants per population) with the backgrounds Ghd7Ghd8Hd1, Ghd7Ghd8hd1 and Ghd7ghd8Hd1 were also grown in Lingshui in winter 2017. In addition, four plants of each fourgene homozygous combination were grown in the field to implement a short-day treatment with a day length of $11 \mathrm{~h}$ and darkness of $13 \mathrm{~h}$ in the summer of 2018. A set of plants from these genotypes were planted in the same field at the same density under NLD conditions and served as the control group.

\section{DNA extraction, polymerase chain reaction and genotyping}

At the tillering stage, leaf blades were collected for DNA extraction using a modified cetyl-trimethyl ammonium bromide (CTAB) method (Murray and Thompson 1980). Genomic DNA was amplified using rTaq polymerase from TaKaRa in Buffer I according to the manufacturer's indications. For each PCR reaction, DNA was initially incubated for 5 minutes at $95^{\circ} \mathrm{C}$, followed by 35 cycles of amplification $\left(95^{\circ} \mathrm{C}\right.$ for $30 \mathrm{~s}, 58^{\circ} \mathrm{C}$ for $30 \mathrm{~s}$ and $72{ }^{\circ} \mathrm{C}$ for $30 \mathrm{~s}$ ). The simple sequence repeat (SSR) marker MRG4436, which is tightly linked to Ghd7, and the functional markers Z9M, InDel37 and S56 designed from Ghd8, PRR37 and Hd1 (Additional file 1: Table S1), respectively, were used to genotype the individuals of all populations and NILs. All markers used for genotyping are listed in (Additional file 1: Table S7).

\section{RNA extraction and qRT-PCR analysis}

Seedlings were grown in a seedbed under NLD conditions for 30 days and were subsequently transplanted to a plot in the field for the short-day treatment (started on the 11th of June, light treatment from 7:00 am to 6:00 pm every day). After treatment for 15 days (from the 11 th to 26th of June), the young leaves in the short-day treatment and control group (treated with LD condition, i.e., more than $14 \mathrm{~h}$ day length per day from the 11 th to 26th of June) were collected at 9:00 am for RNA extraction. For each genotype, leaves from three different individuals were collected as biological replicates. Total RNA was extracted using TRIzol reagent (TransGen Biotech, Beijing) and treated with DNase I (Invitrogen, USA). cDNA was synthesized from $3 \mu \mathrm{g}$ of RNA using SuperScript III Reverse Transcriptase (Invitrogen, USA). The quantitative analysis of gene expression was performed with SYBR Premix ExTaq reagent (TaKaRa, Dalian) on the ABI ViiA7 Real-time PCR System (Applied Biosystems, USA). The data were analyzed using the relative quantification method. The primers used for real-time PCR are listed in (Additional file 1: Table S7).

\section{Trait measurement and data analysis}

Heading date was individually scored as the number of days from sowing to the emergence of the first panicle on the plant. The total number of spikelets per plant was measured by the Yield Traits Scorer (Yang et al. 2014). The number of spikelets per panicle (SPP) of each homozygous combination line was recorded as the total number of spikelets divided by the number of panicles. The comparison between genotypes was performed by Student's $t$-test. To verify the existence of high order genetic interactions, the three-way ANOVA or factorial ANOVA were performed under the condition of fixation of the allele at the fourth gene. The statistical significance of three-way interactions was evaluated by a general liner model (GLM) using the program STATISTICA 8.0 (Statsoft 1995).

\section{Results}

\section{Composition of major heading date genes in ZS97}

Our previous studies confirmed that ZS97 carried a functional allele of $H d 1$ and nonfunctional alleles of Ghd7, Ghd8 and PRR37/Ghd7.1 (Xue et al. 2008; Yan et al. 2011; Yan et al. 2013; Zhang et al. 2017). To clarify the genetic background on heading date, the coding sequences and functional nucleotide polymorphisms of other 10 major flowering genes were downloaded from the reference genome of ZS97 and Rice SNP-Seek Database, respectively (Alexandrov et al. 2015; Song et al. 2018; Wang et al. 2018). Alignment of coding sequence were used to compare allele identity between ZS97 and varieties used in previous studies (Additional file 1: Table S3). Alleles of DTH3/OsMADS5O and Hd6 were the same as the one carried by Dianjingyou 1 and Kasalath, respectively, which were the functional alleles (Takahashi et al. 2001; Lee et al. 2004; Bian et al. 2011). The haplotypes of $H d 16 / E L 1, H d 3 a$ and Ehd1 were identified as Type 4, Type 3 and Type 6, respectively, which were also confirmed as the functional types (Takahashi et al. 2009; Hori et al. 2013; Kwon et al. 2014). Allele of Ehd4 in ZS97 was the same as the 93-11 haplotype, Hap_2, which was a weak functional allele demonstrated by transgenic verification (Gao et al. 2013). The haplotype 
of $H d 17$ in ZS97 was consistent with that in Koshihikari, which was a weak allele compared with Nipponbare (Matsubara et al. 2012). Allele of Hd18 in ZS97 was the same as that in Hayamasari, acted as a weak allele (Shibaya et al. 2016). The haplotype of DTH2 in ZS97 was consistent with Group A1, which was a nonfunctional allele (Wu et al. 2013). RFT1 in ZS97 belonged to Type IIb with E105K variation and also exhibited a loss of function (Zhao et al. 2015).

\section{The genetic interactions among Ghd7, Ghd8, PRR37 and} Hd1 under NLD conditions

The NIL- $F_{1}$ plant carrying heterozygous alleles at these four genes (Additional file 1: Figure S1a) was genotyped by the RICE6K SNP array. More than $90 \%$ of the NIL-F plant background was consistent with ZS97, but the segments harboring Ghd7, Ghd8, PRR37 and $H d 1$ were heterozygous. The segments harboring other 10 flowering gene regions were fixed with ZS97 genotype in the NIL$\mathrm{F}_{1}$ plant (Additional file 1: Figure S1b). In the NIL- $\mathrm{F}_{2}$ population, large variation in heading date was observed, ranging from 65 days to no heading after 160 days under NLD conditions (Fig. 1a). For convenience, 160 days was recorded as the heading date of these non-heading plants. Two-way and three-way ANOVA separately showed that all 6 pairs of digenic interactions and 4 trigenic interactions were highly significant (Additional file 1: Table S4). Four-way ANOVA revealed that the tetragenic interaction among these four genes was also highly significant (Additional file 1: Table S4). To better understand the four-way interaction, we classified the populations into three subpopulations based on $H d 1$ genotypes: homozygous $H d 1$, heterozygous $H d 1\left(H d 1^{H}\right)$ and homozygous $h d 1$. A significant threeway interaction was detected among Ghd7, Ghd8 and PRR37 at $P<1.0 \mathrm{E}-10$ in both the $H d 1$ and $H d 1^{H}$ backgrounds and at $P=6.9 \mathrm{E}-04$ in the $h d 1$ background (Additional file 1: Figure S2a-c; Table 1). Additionally, all digenic interactions were detected among Ghd7, Ghd8 and PRR37. The Ghd7 by Ghd8 interaction contributed more to heading date variation than the other digenic interactions. The square of this interaction accounted for $5.9 \%$ and $5.8 \%$ of the total sum-of-squares in the $H d 1$ and $H d 1^{H}$ backgrounds, respectively, and $5.8 \%$ of that in the nonfunctional $h d 1$ background (Table 1). The main effects of $G h d 7$, Ghd8 and their digenic interaction effects explained more than $70 \%$ of the variation in heading date in both the $H d 1$ and $H d 1^{H}$ backgrounds. The genetic square of PRR37 accounted for $17.0 \%$ of the total sum-of-squares in the $h d 1$ background, which was much larger than that observed in the $H d 1$ and $H d 1^{H}$ backgrounds (Table 1 ). Taken together, these results revealed that a strong trigenic interaction existed among Ghd7, Ghd8 and PRR37 regardless of the genotype of $H d 1$, and the interaction between Ghd7 and Ghd8 showed the strongest digenic interaction among these three genes under NLD conditions.

\section{The genetic interactions among Ghd7, Ghd8, PRR37 and Hd1 under NSD conditions}

The heading date variation of NIL- $\mathrm{F}_{3}$ population exhibited a continuous distribution ranging from 82 days to 124 days (Fig. 1b). Accordingly, all digenic and trigenic interactions (except the Ghd8 by PRR37 by $H d 1$ interaction) among these four genes were significant under NSD conditions (Additional file 1: Table S4). A significant tetragenic interaction was also observed in the NIL$\mathrm{F}_{3}$ (Additional file 1: Table S4). Following the analysis performed for NLD conditions, this population were also classified into 3 classes according to $H d 1$ genotypes. Significant interactions were identified among Ghd7, Ghd8 and PRR37 in the $h d 1, H d 1^{H}$ and $H d 1$ backgrounds (Additional file 1: Figure S2d-f; Table 2). However, the
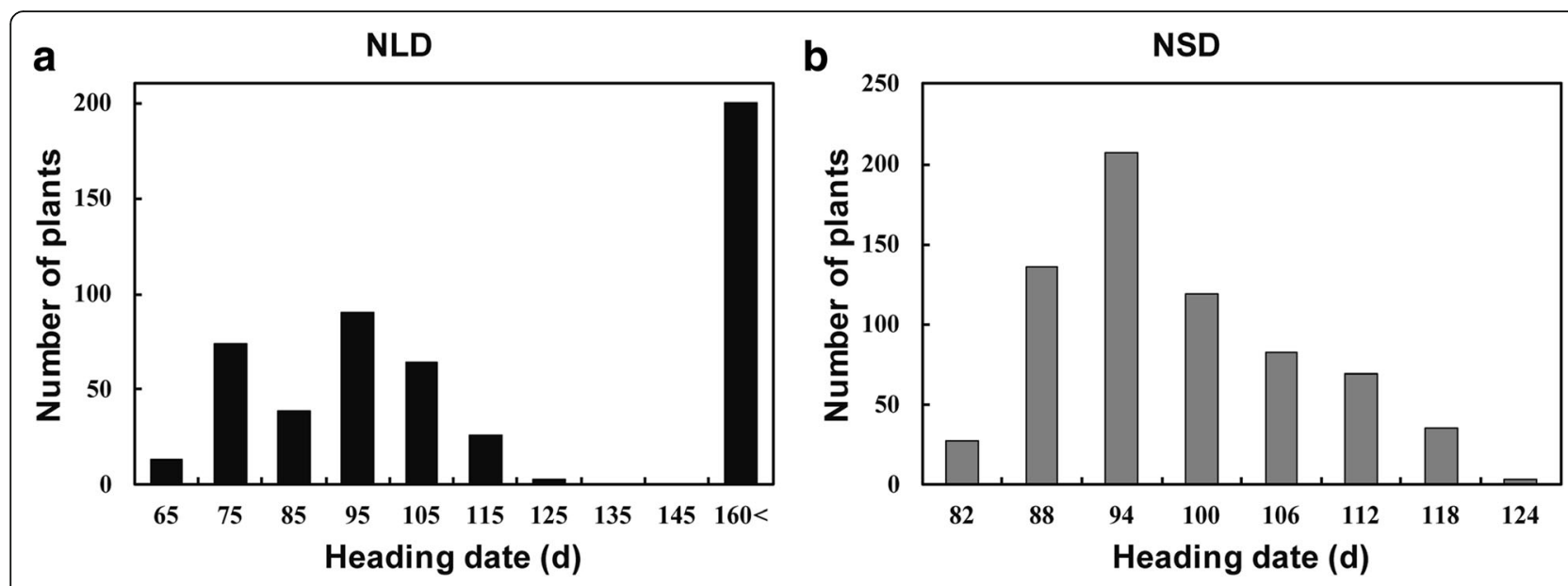

Fig. 1 The heading date distribution of 4-gene segregating populations under NLD and NSD conditions. The heading date distribution of NIL-F 2 population under NLD conditions (a) and of NIL-F3 population under NSD conditions (b). "160<", non-heading after 160 days from sowing 
Table 1 Three-way ANOVA analysis of Ghd7, Ghd8 and PRR37 in NIL-F $F_{2}$ population under NLD conditions

\begin{tabular}{|c|c|c|c|c|c|c|c|c|c|c|}
\hline \multirow[t]{2}{*}{ Effect } & \multirow[t]{2}{*}{$\mathrm{D}$} & \multicolumn{3}{|c|}{$h d 1$ ( $\left.n=138 ;{ }^{a} 74 d-128 d\right)$} & \multicolumn{3}{|c|}{$H d 1^{H}\left(n=242 ; 67 d-{ }^{b} 160 d\right)$} & \multicolumn{3}{|c|}{ Hd1 $(n=129 ; 64 d-160 d)$} \\
\hline & & $\bar{F}$ & $P$ & $\overline{G: T ~(\%)}$ & $\bar{F}$ & $P$ & G:T (\%) & $\bar{F}$ & $P$ & G:T (\%) \\
\hline Ghd7 & 2 & 787.7 & $<1.0 \mathrm{E}-10$ & 28.6 & 12145.5 & $<1.0 \mathrm{E}-10$ & 29.6 & 9627.4 & $<1.0 \mathrm{E}-10$ & 31.9 \\
\hline Ghd8 & 2 & 800.5 & $<1.0 \mathrm{E}-10$ & 29.1 & 14400.5 & $<1.0 \mathrm{E}-10$ & 35.1 & 11278.0 & $<1.0 \mathrm{E}-10$ & 37.4 \\
\hline PRR37 & 2 & 468.3 & $<1.0 \mathrm{E}-10$ & 17.0 & 747.6 & $<1.0 \mathrm{E}-10$ & 1.8 & 799.1 & $<1.0 \mathrm{E}-10$ & 2.6 \\
\hline Ghd7 by Ghd8 & 4 & 80.1 & $<1.0 \mathrm{E}-10$ & 5.8 & 1183.4 & $<1.0 \mathrm{E}-10$ & 5.8 & 893.3 & $<1.0 \mathrm{E}-10$ & 5.9 \\
\hline Ghd7 by PRR37 & 4 & 9.6 & 1.1E-06 & 0.7 & 116.5 & $<1.0 \mathrm{E}-10$ & 0.6 & 239.9 & $<1.0 \mathrm{E}-10$ & 1.6 \\
\hline Ghd8 by PRR37 & 4 & 21.1 & $<1.0 \mathrm{E}-10$ & 1.5 & 34.8 & $<1.0 \mathrm{E}-10$ & 0.2 & 31.1 & $<1.0 \mathrm{E}-10$ & 0.2 \\
\hline Ghd7 by Ghd8 by PRR37 & 8 & 3.7 & $6.9 \mathrm{E}-04$ & 0.5 & 88.5 & $<1.0 \mathrm{E}-10$ & 0.9 & 63.8 & $<1.0 \mathrm{E}-10$ & 0.8 \\
\hline
\end{tabular}

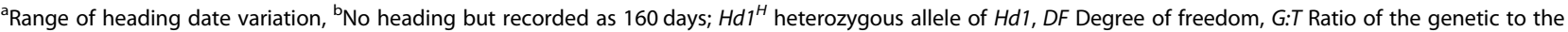
total of sum-of-squares

digenic interactions among these three genes were different from those detected under NLD conditions. The Ghd7 by PRR37 interaction contributed much more to heading date variation than the other two digenic interactions in the functional $H d 1$ backgrounds, in which the genetic square accounted for $20.3 \%$ and $20.4 \%$ of the total sum-of-squares in the $H d 1$ and $H d 1^{H}$ backgrounds, respectively (Table 2). Notably, the effect of Ghd7 on heading date was the strongest under NSD conditions, explaining $58 \%, 21.7 \%$ and $29.1 \%$ of the variation in the $h d 1, H d 1^{H}$ and $H d 1$ backgrounds, respectively (Table 2 ). These results indicated that Ghd7, Ghd8 and PRR37 interacted under NSD conditions and the Ghd7 by PRR37 interaction showed the strongest epistatic effect among the digenic interactions in the functional $H d 1$ backgrounds.

\section{PRR37 acts as a heading date suppressor under NLD conditions}

To estimate the additive and dominance effects of PRR37 in different genetic backgrounds under NLD conditions, we developed 8 PRR37-segregating populations (NIL- $\mathrm{F}_{4}$ ) with different homozygous combinations of the other three genes. The NIL-F 4 population with the Ghd7Ghd8Hd1 background did not head even after October 24th, when the low temperature is unfavorable to rice growing in Wuhan (Fig. 2a). Therefore, no data were used to evaluate the genetic effect of PRR37 in this population (Table 3 ). We merged the $8 \mathrm{NIL}^{-\mathrm{F}_{4}}$ populations for interaction analysis because these populations shared similar genetic background and were grown in the same condition. All digenic and trigenic interactions or even tetragenic interaction among these four genes were also highly significant (Additional file 1: Figure S3a-c; Table S5). To confirm whether PRR37 also delayed rice heading in the Ghd7Ghd8Hd1 background, we took the young panicles of the main stems of the two homozygous combinations, namely, Ghd7Ghd8Hd1PRR37 and Ghd7Ghd8Hd1prr37, on September 30th and compared their lengths (Fig. 2b). The young panicle length of Ghd7Ghd8Hd1PRR37 $(0.87 \mathrm{~cm})$ was significantly shorter than that of Ghd7Ghd8Hd1prr37 $(1.55 \mathrm{~cm})$, which suggested that PRR37 suppressed heading in the Ghd7Ghd8Hd1 background (Fig. 2c). The additive effect of PRR37 in the other 7 populations ranged from 5.6-19.4 days, indicating that PRR37 always plays as a suppressor of heading date in these backgrounds under NLD conditions (Table 3). The dominance effects and degrees of dominance of PRR37 ranged from 2.4-10.7 days and from $0.28-0.93$, respectively (Table 3 ). Accordingly, we observed large heading date variations in the ghd7Ghd8Hd1 and Ghd7Ghd8hd1 backgrounds, ranging from 69 to 115 days and from 94 to 127 days, respectively (Table 3 ). The effects

Table 2 Three-way ANOVA analysis of Ghd7, Ghd8 and PRR37 in NIL-F population under NSD conditions

\begin{tabular}{|c|c|c|c|c|c|c|c|c|c|c|}
\hline \multirow[t]{2}{*}{ Effect } & \multirow[t]{2}{*}{$\mathrm{D}$} & \multicolumn{3}{|c|}{$h d 1\left(n=165 ;{ }^{a} 96 d-127 d\right)$} & \multicolumn{3}{|c|}{$\mathrm{Hdl}^{H}(n=339 ; 86 \mathrm{~d}-118 \mathrm{~d})$} & \multicolumn{3}{|c|}{$\operatorname{Hd1}(n=175 ; 82 d-122 d)$} \\
\hline & & $\mathrm{F}$ & $P$ & G:T (\%) & $\mathrm{F}$ & $P$ & G:T (\%) & $\mathrm{F}$ & $P$ & G:T (\%) \\
\hline Ghd7 & 2 & 371.1 & $<1.0 \mathrm{E}-10$ & 58.0 & 144.5 & $<1.0 \mathrm{E}-10$ & 21.7 & 107.0 & $<1.0 \mathrm{E}-10$ & 29.1 \\
\hline Ghd8 & 2 & 11.2 & $3.2 \mathrm{E}-05$ & 1.7 & 8.4 & $2.9 \mathrm{E}-04$ & 1.3 & 36.2 & $<1.0 \mathrm{E}-10$ & 9.8 \\
\hline PRR37 & 2 & 16.9 & $2.6 \mathrm{E}-07$ & 2.6 & 55.7 & $<1.0 \mathrm{E}-10$ & 8.4 & 10.9 & 4.0E-05 & 2.9 \\
\hline Ghd7 by Ghd8 & 4 & 23.1 & $<1.0 \mathrm{E}-10$ & 7.2 & 9.6 & $2.4 \mathrm{E}-07$ & 2.9 & 14.5 & $5.4 \mathrm{E}-10$ & 7.9 \\
\hline Ghd7 by PRR37 & 4 & 13.0 & $5.0 \mathrm{E}-09$ & 4.1 & 67.9 & $<1.0 \mathrm{E}-10$ & 20.4 & 37.4 & $<1.0 \mathrm{E}-10$ & 20.3 \\
\hline Ghd8 by PRR37 & 4 & 15.0 & $3.1 \mathrm{E}-10$ & 4.7 & 19.5 & $<1.0 \mathrm{E}-10$ & 5.9 & 6.5 & 7.6E-05 & 3.5 \\
\hline Ghd7 by Ghd8 by PRR37 & 8 & 3.0 & 4.1E-03 & 1.9 & 8.3 & $3.1 \mathrm{E}-10$ & 5.0 & 4.4 & 9.3E-05 & 4.8 \\
\hline
\end{tabular}

${ }^{a}$ Range of heading date variation; $\mathrm{Hdl}^{\mathrm{H}}$ Heterozygous allele of $\mathrm{Hd1}$, DF Degree of freedom, G:T Ratio of the genetic to the total of sum-of-squares 


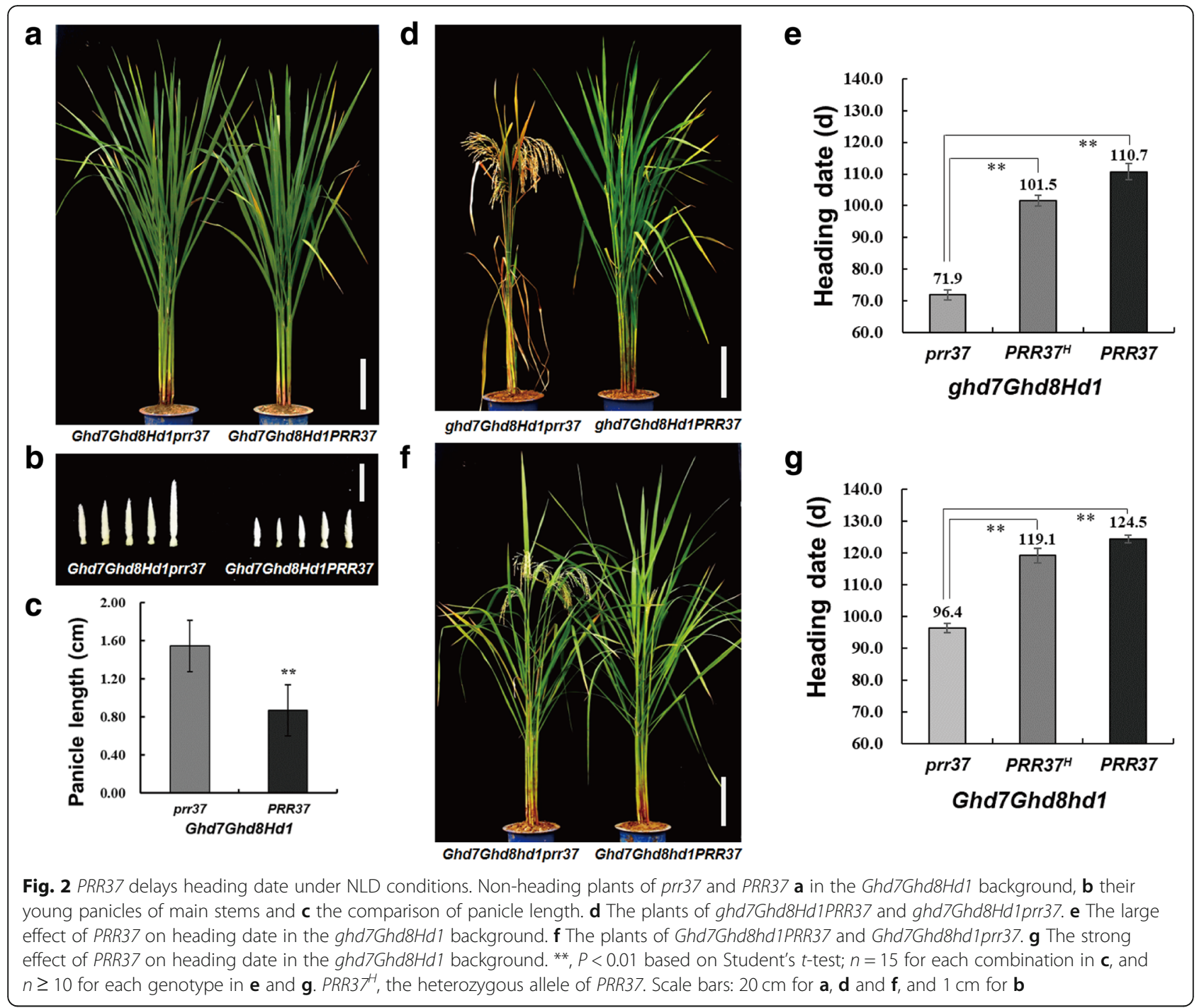

Table 3 The genetic effects of PRR37 on heading date in 8 NIL-F 4 populations under NLD conditions

\begin{tabular}{|c|c|c|c|c|c|c|c|c|}
\hline \multirow[t]{2}{*}{ Background } & \multirow[t]{2}{*}{ Size } & \multicolumn{7}{|c|}{ Heading date (d) } \\
\hline & & Range & prr37 & $P R R 37^{H}$ & PRR37 & $A$ & $\mathrm{D}$ & $|\mathrm{D} / \mathrm{A}|$ \\
\hline ghd7ghd8hd1 & 47 & $70-88$ & $71.0 \pm 1.7$ & $81.3 \pm 2.3$ & $84.7 \pm 1.8$ & 6.8 & 3.4 & 0.50 \\
\hline ghd7Ghd8hd1 & 51 & $74-97$ & $75.7 \pm 1.2$ & $84.9 \pm 1.8$ & $92.7 \pm 2.3$ & 8.5 & NS & \\
\hline Ghd7ghd8hd1 & 52 & 77-93 & $79.1 \pm 1.8$ & $87.0 \pm 1.6$ & $90.2 \pm 1.8$ & 5.6 & 2.4 & 0.43 \\
\hline Ghd7Ghd8hd1 & 59 & $94-127$ & $96.4 \pm 1.4$ & $119.1 \pm 2.4$ & $124.5 \pm 1.2$ & 14.0 & 8.7 & 0.62 \\
\hline ghd7ghd8Hd1 & 47 & $59-82$ & $61.5 \pm 1.5$ & $73.2 \pm 2.7$ & $79.8 \pm 1.7$ & 9.1 & 2.5 & 0.28 \\
\hline ghd7Ghd8Hd1 & 47 & 69-115 & $71.9 \pm 1.5$ & $101.5 \pm 1.7$ & $110.7 \pm 2.5$ & 19.4 & 10.2 & 0.53 \\
\hline Ghd7ghd8Hd1 & 58 & 80-108 & $81.6 \pm 1.3$ & $103.7 \pm 1.3$ & $104.5 \pm 1.6$ & 11.5 & 10.7 & 0.93 \\
\hline Ghd7Ghd8Hd1 & 60 & $\mathrm{NH}$ & $\mathrm{NH}$ & $\mathrm{NH}$ & $\mathrm{NH}$ & & & \\
\hline
\end{tabular}

Size, the number of plants of segregating population; $P R R 37^{H}$, heterozygous allele of $P R R 37 ; \mathrm{A}$, additive effect; $\mathrm{D}$, dominance effect; $|\mathrm{D} / \mathrm{A}|$, the degree of dominance; NS, no significance; $\mathrm{NH}$, no heading 
of PRR37 on heading date were 38.8 and 28.1 days in these two backgrounds, respectively, which were much larger than that in the ghd7ghd8hd1 background (Fig. 2d-g; Table 3). These results revealed that the large genetic effects of PRR37 on heading date were dependent on the combinations of Ghd7, Ghd8 and $H d 1$.

\section{Alternative functions of $P R R 37$ in repressing or promoting heading under NSD conditions}

Heading dates of these 8 PRR37-segregating populations $\left(\mathrm{NIL}-\mathrm{F}_{4}\right)$ also exhibited a continuous distribution ranging from 95 to 135 days under NSD conditions (Additional file 1: Figure S3d). We merged these 8 populations together for interaction analysis. Accordingly, most of digenic and trigenic interactions and tetragenic interaction among these four genes were significant (Additional file 1: Figure S3e-f; Table S5). The additive effects of PRR37 were 1.8 days, 5.0 days and 2.2 days in the ghd7ghd8hd1, ghd7ghd8Hd1 and ghd7Ghd8Hd1 backgrounds, respectively (Table 4), indicating that PRR37 acted as a flowering suppressor in these three backgrounds. However, the effect on delaying heading date was much smaller than that observed under NLD conditions. The genetic effect of PRR37 disappeared in the ghd7Ghd8hd1 and Ghd7ghd8hd1 backgrounds. Interestingly, a converse effect of PRR37 on heading date was observed in the Ghd7ghd8Hd1, Ghd7Ghd8Hd1 and Ghd7Ghd8hd1 backgrounds comparing with that observed under NLD conditions. The additive effects of PRR37 in these three backgrounds were -2.0 days, -9.9 days and -3.7 days, respectively (Table 4). Therefore, it seemed that PRR37 acted as a heading activator in these three backgrounds. Three additional PRR37-segregating populations with the Ghd7ghd8Hd1, Ghd7Ghd8Hd1 and Ghd7Ghd8hd1 backgrounds were used to verify this finding. Compared to prr37, PRR37 promoted rice heading by 4.8 days, 18.0 days and 5.3 days in these three backgrounds, respectively (Fig. 3a-c). In addition, PRR37 promoted heading by 3.0 days, 12.0 days and 18.2 days in these three backgrounds in the 11-h light treatment, respectively (Fig. 3d-f). These data clearly demonstrated that PRR37acted as a heading activator in the Ghd7ghd8Hd1, Ghd7Ghd8Hd1 and Ghd7Ghd8hd1 backgrounds under NSD conditions. The dominance effects and degrees of dominance of PRR37 in these 8 populations ranged from 7.3 to 1.9 days and from 0.38 to 0.88 , respectively (Table 4 ), suggesting that the genetic effects of PRR37 were largely influenced by the genetic background.

\section{Transcriptional analysis of Ehd1 and $\mathrm{Hd} 3 \mathrm{a}$ in the Ghd7ghd8Hd1, Ghd7Ghd8Hd1 and Ghd7Ghd8hd1 backgrounds}

Considering that PRR37 has an alternative function in these three backgrounds under different day-length conditions, the expression of PRR37 downstream genes, Ehd1 and $H d 3 a$, was compared between prr37 and PRR37 in these three backgrounds under LD and SD conditions, respectively. The relative expression levels of Ehd1 and Hd3a in Ghd7ghd8Hd1PRR37 and Ghd7Ghd8hd1PRR37 genotypes decreased under LD conditions but increased under SD conditions compared with those in Ghd7ghd8Hd1prr37 and Ghd7Ghd8hd1prr37, respectively (Fig. 4). The expression of Ehd1 and $H d 3 a$ showed no significant difference between prr37 and PRR37 in the Ghd7Ghd8Hd1 background under LD conditions but increased with the presence of PRR37 under SD conditions (Fig. 4). These results indicated that PRR37 promoted the expression of Ehd 1 and $H d 3 a$ in these three backgrounds under SD conditions, resulting in an early heading date. In contrast, PRR37 delayed rice heading in the $g h d 7 g h d 8 H d 1$ background by repressing the expression of Ehd1 and Hd3a under both LD and SD conditions (Additional file 1: Figure S4).

\section{Correlation between heading date and SPP under NLD and NSD conditions}

We identified the relationship between heading date and SPP on the basis of performance of 16 homozygous 4-

Table 4 The genetic effects of PRR37 on heading date in 8 NIL-F 4 populations under NSD conditions

\begin{tabular}{|c|c|c|c|c|c|c|c|c|}
\hline \multirow[t]{2}{*}{ Background } & \multirow[t]{2}{*}{ Size } & \multicolumn{7}{|c|}{ Heading date $(\mathrm{d})$} \\
\hline & & Range & prr37 & PRR37 $7^{H}$ & PRR37 & A & D & $|D / A|$ \\
\hline ghd7ghd8hd1 & 40 & $112-122$ & $114.9 \pm 1.5$ & $118.2 \pm 1.5$ & $118.4 \pm 2.0$ & 1.8 & 1.6 & 0.88 \\
\hline ghd7Ghd8hd1 & 39 & $110-117$ & $114.9 \pm 1.6$ & $114.7 \pm 1.0$ & $113.5 \pm 1.6$ & NS & NS & \\
\hline Ghd7ghd8hd1 & 38 & $120-126$ & $123.2 \pm 0.8$ & $123.3 \pm 1.9$ & $123.7 \pm 1.0$ & NS & NS & \\
\hline Ghd7Ghd8hd1 & 40 & $123-135$ & $133.4 \pm 1.3$ & $127.1 \pm 1.5$ & $126.0 \pm 1.2$ & -3.7 & -2.6 & 0.71 \\
\hline ghd7ghd8Hd1 & 39 & $97-112$ & $100.0 \pm 2.1$ & $106.9 \pm 3.0$ & $110.0 \pm 1.4$ & 5.0 & 1.9 & 0.38 \\
\hline ghd7Ghd8Hd1 & 40 & $102-112$ & $104.5 \pm 1.9$ & $108.1 \pm 1.7$ & $108.9 \pm 1.6$ & 2.2 & 1.4 & 0.64 \\
\hline Ghd7ghd8Hd1 & 38 & $108-116$ & $113.3 \pm 1.6$ & $111.9 \pm 1.5$ & $109.3 \pm 1.3$ & -2.0 & NS & \\
\hline Ghd7Ghd8Hd1 & 40 & $113-136$ & $133.4 \pm 1.7$ & $116.2 \pm 2.3$ & $113.6 \pm 1.1$ & -9.9 & -7.3 & 0.74 \\
\hline
\end{tabular}

Size, the number of plants of segregating population; $P R R 37^{H}$, heterozygous allele of PRR37; Negative value indicates the functional allele of $P R R 37$ promotes rice heading. $A$, additive effect; $D$, dominance effect; $|D / A|$, the degree of dominance; NS, no significance 


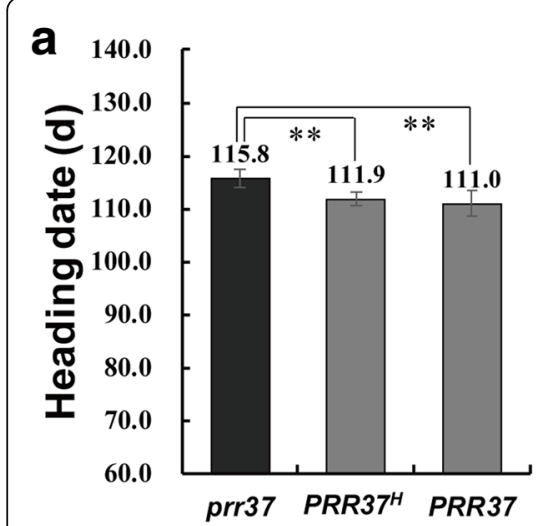

Ghd7ghd8Hd1
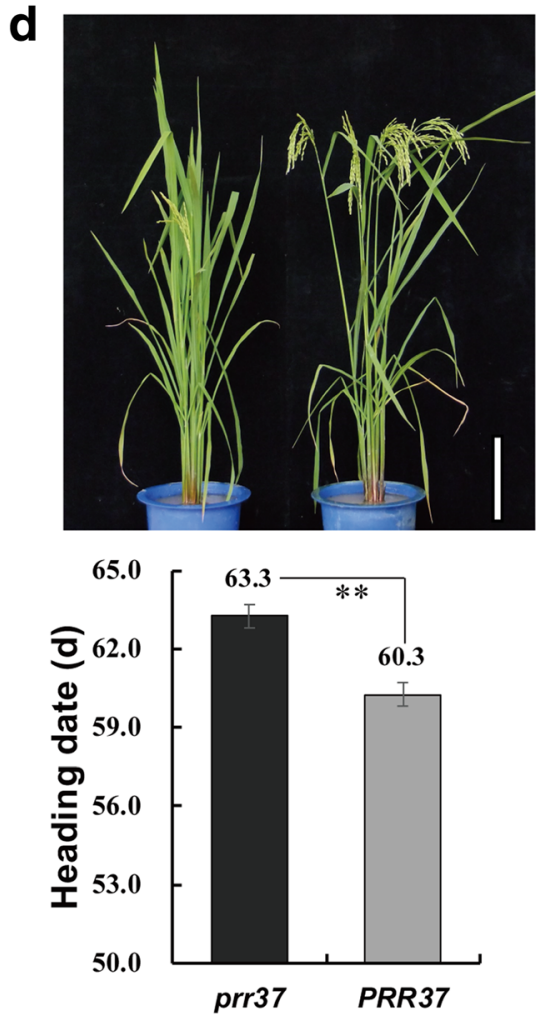

Ghd7ghd8Hd1

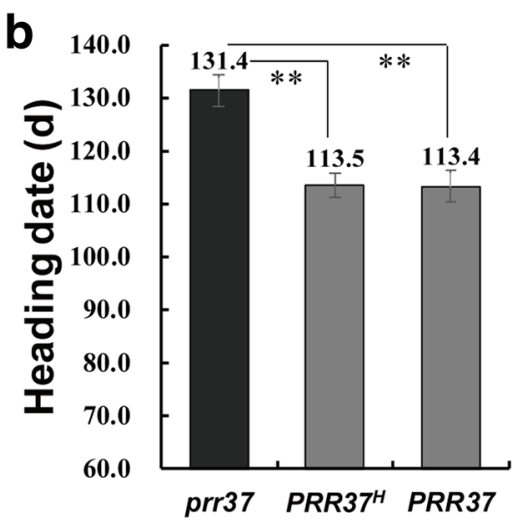

Ghd7Ghd8Hd1
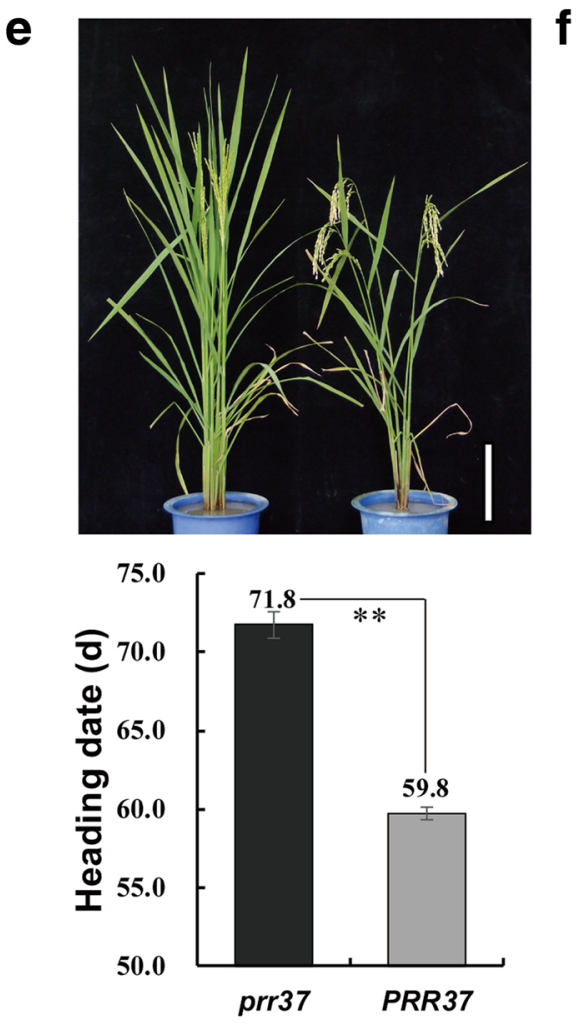

Ghd7Ghd8Hd1

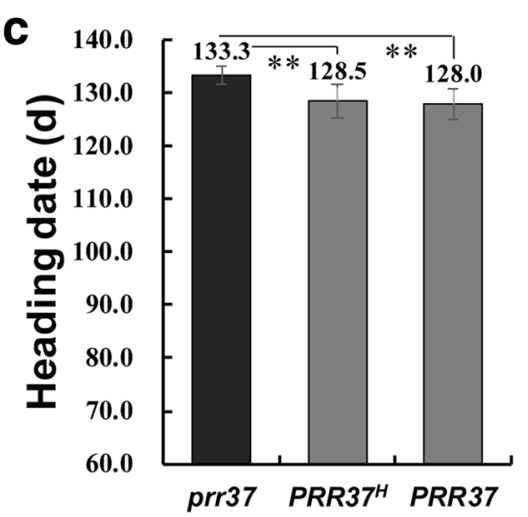

Ghd7Ghd8hd1
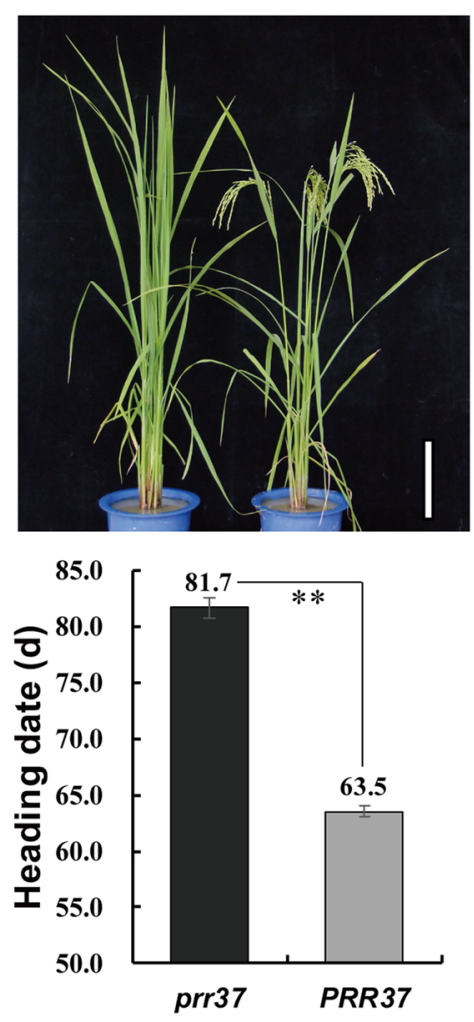

Ghd7Ghd8hd1

Fig. 3 PRR37 promotes rice heading in the specific backgrounds under NSD and SD conditions. Comparisons of heading date among different alleles of PRR37 in the backgrounds Ghd7ghd8Hd1 $\mathbf{a}$, Ghd7Ghd8Hd1 $\mathbf{b}$ and Ghd7Ghd8hd1 $\mathbf{c}$ under NSD conditions ( $\mathrm{n} \geq 10$ for each combination). d-f, Pictures (top) and heading dates (bottom) of prr37 and PRR37 in each corresponding background under SD conditions ( $n=4$ for each combination). ${ }^{* *}, P<0.01$ based on Student's $t$-test. $P R R 37^{H}$, the heterozygous allele of $P R R 37$. Scale bars: $20 \mathrm{~cm}$ for $\mathbf{d}, \mathbf{e}$ and $\mathbf{f}$

gene combinations (Fig. 5; Additional file 1: Table S6). Under NLD conditions, the heading date of these 16 combinations exhibited a continuous distribution ranging from 60 days to 130 days except for the two nonheading combinations Ghd7Ghd8PRR37Hd1 and Ghd7Ghd8prr37Hd1. The earliest heading combination was $g h d 7 g h d 8 p r r 37 H d 1$ with 60.8 days, which was the ZS97 genotype (Fig. 5a; Additional file 1: Table S6).
Unexpectedly, SPP of these 14 combinations showed an inverse correlation with heading date. The SPP increased with later heading dates when the heading date was earlier than 90 days, while the SPP decreased with later heading dates when heading date was after 90 days (Fig. 5b). The curve-fitting plots of heading date with SPP under NLD conditions also revealed the inverse correlation with an inflection point at 90.0 days (Fig. 5c). The 

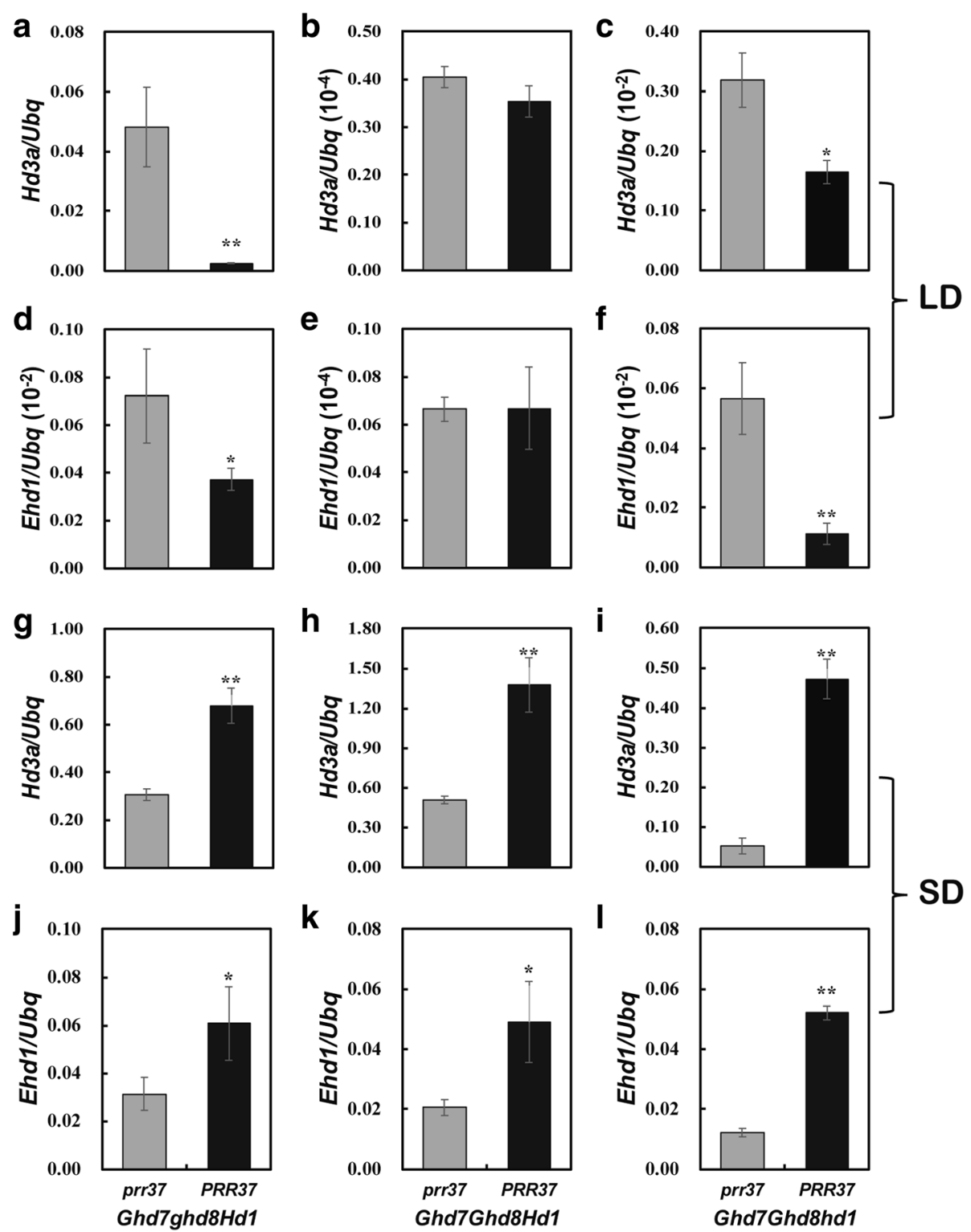

Fig. 4 Transcript levels of Hd3a and Ehd1 between prr37 and PRR37 under LD and SD conditions. Relative expression levels of Hd3a and Ehd1 between prr37 and PRR37 in the Ghd7ghd8Hd1 (a, d), Ghd7Ghd8Hd1 (b, e) and Ghd7Ghd8hd1 (c, f) backgrounds under LD conditions, respectively; Relative expression levels of Hd3a and Ehd1 between prr37 and PRR37 in the Ghd7ghd8Hd1 ( $\mathbf{g}, \mathbf{j}$ ), Ghd7Ghd8Hd1 (h, k) and Ghd7Ghd8hd1 (i, l) backgrounds under SD conditions, respectively. ${ }^{*}$ and ${ }^{* *}, P<0.05$ and $P<0.01$ based on Student's $t$-test, respectively

combination $g h d 7 g h d 8 P R R 37 h d 1$ had the most SPP, with $199.9 \pm 7.3$ under NLD conditions, and the second most was Ghd7ghd8prr37Hd1, with $184.1 \pm 9.8$ (Additional file 1: Table S6). Under NSD conditions, the heading date of the 16 combinations also showed a continuous distribution with a range from 98 days to 132 days. The combination with the earliest heading date was also the ZS97 genotype, ghd7ghd8prr37Hd1, at 98.7 days, while the combination with the latest heading date was Ghd7Ghd8prr37hd1, at 131.8 days (Fig. 5d; Additional file 1: Table S6). The SPP of these 16 combinations increased with the later heading dates, indicating that SPP was positively correlated with heading date under NSD conditions (Fig. 5e-f).

\section{Discussion}

Ghd7, Ghd8, PRR37/Ghd7.1 and Hd1 are all photoperiod sensitive genes that respond to day-length changes and play important roles in rice adaptation to high latitude regions (Yano et al. 2000; Xue et al. 2008; Yan et al. 2011; Liu et al. 2013; Koo et al. 2013). Their combinations also largely determine the adaptation and yield potential of rice cultivars. Loss-of-function allele combination $(\mathrm{NNN})$ and pre-existing strong allele combination 


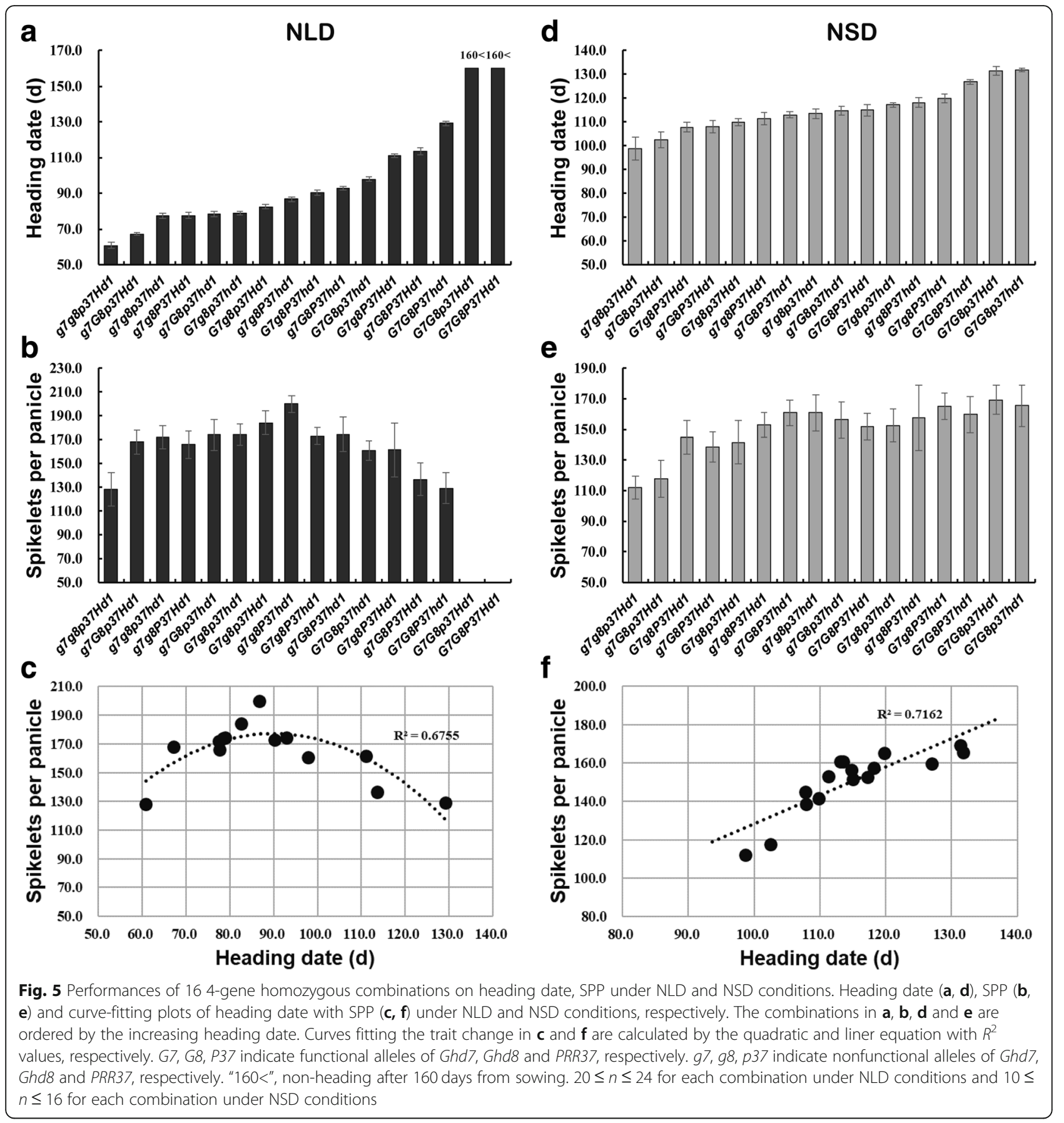

(SSF) of Ghd7, Ghd 8 and $H d 1$ allow rice cultivars to adapt to temperate and tropical regions, respectively (Zhang et al. 2015a). Loss-of-function alleles of Ghd7, PRR37/DTH7 and $H d 1$ contributed to early rice heading dates in the northern regions of northeast China, while functional alleles delayed heading in the southern regions of northeast China, indicating that divergent alleles of these three genes largely determined rice adaptation in northeast China (Ye et al. 2018). In this study, the combinations of Ghd7, Ghd8, PRR37 and Hd1 in ZS97 background exhibited stronger photoperiod sensitivity under NLD conditions than under NSD conditions. Significant digenic, trigenic or even tetragenic interactions of these four genes were detected under both conditions (Additional file 1: Table S4), but the significance detected under NLD conditions was much greater than that detected under NSD conditions, where the effects of Ghd7, Ghd8 and PRR37 were decreased. The OsHAPL1DTH8-Hd1 complex acts as a transcriptional regulator of heading date by interacting with the HAP complex and GTFs (Zhu et al. 2017). Ghd8/DTH8 encodes a HAP3 
subunit, which can form a multicomplex with HAP2 and HAP5 (Thirumurugan et al. 2008). Ghd7, PRR37 and Hd1 encode transcription factors containing CCT domains, which are similar to HAP2 and responsible for DNA binding and protein-protein interaction (Wenkel et al. 2006; Thirumurugan et al. 2008). Thus, interactions among these genes probably indicate physical interactions among their encoding proteins or between proteins (transcriptional factors) and DNA elements (gene promoters). In addition, only strong functional and nonfunctional alleles were taken into consideration in this study. The heading date of these 16 four-gene combinations showed a continuous distribution with a range of 60-130 days and no heading under NLD conditions in Wuhan and a range of 98-132 days under NSD conditions in Hainan (Fig. 5). In nature, there are more diverse alleles for each gene (Koo et al. 2013; Zhang et al. 2015a). It is expected that different gene combinations will have similar heading dates due to the comprehensive effect of single gene and interaction effects. A better understanding of these four major flowering genes will aid in breeding design for developing cultivars for local rice production. It is noticed that these findings are derived from typical Xian (indica) cultivar, ZS97. It is not clear whether similar results would be obtained in Geng (japonica), which is worth testing in the future.

Grain yield is positively correlated with heading date, especially in low latitude areas where the temperature is warm year-round (Gao et al. 2014; Li et al. 2018). In this study, due to continuously high temperature stress during the rice flowering stage in Wuhan, the seed setting rates were significantly decreased; therefore, we analyzed the relationship between heading date and SPP instead of that between heading date and grain yield. The SPP is consistently and positively correlated with heading date under NSD conditions. Nevertheless, The SPP exhibited an inverse correlation with heading date under NLD conditions. The SPP increased with increasing days from sowing to heading when the heading date was earlier than 90 days, while it decreased with increasing days when the heading date was later than 90 days. Based on this finding, optimized combinations can be suggested for local regions to maximize rice production in indica varieties. For example, varieties with the Ghd7Ghd8prr37Hd1 and Ghd7Ghd8prr37hd1 combinations will produce more grains in low latitude regions (tropical regions) with short-day and warm conditions such as Hainan. In subtropical regions like Wuhan, the ghd7ghd8PRR37hd1 and Ghd7ghd8prr37Hd1 combinations will have the highest yield potential. In this study, the set of materials was grown at only two locations. If they were tested in multiple diverse ecological areas, the favorable gene combinations could be defined for each area.

Previous studies showed that PRR37 inhibited heading date under LD conditions but seemed to have no effect under SD conditions (Koo et al. 2013; Liu et al. 2013; Gao et al. 2014). However, in this study, PRR37 delayed rice heading in the ghd7ghd8hd1, ghd7ghd8Hd1 and ghd7Ghd8Hd1 backgrounds but significantly promoted heading in the Ghd7Ghd8hd1, Ghd7Ghd8Hd1 and Ghd7ghd8Hd1 backgrounds under NSD conditions (Fig. 3; Table 4), which clearly demonstrated that PRR37 had alternative functions under SD conditions. PRR37 suppressed heading date by inhibiting the expression of its downstream genes Ehd1 and $H d 3 a$ under LD conditions. In contrast, PRR37 acted as an activator of rice heading by promoting Ehd1 and $H d 3 a$ expression in the Ghd7Ghd8hd1, Ghd7Ghd8Hd1 and Ghd7ghd8Hd1 backgrounds under SD conditions (Fig. 4). All these three backgrounds had functional allele of $G h d 7$, indicating that Ghd7 played an essential role in the function inversion of PRR37. However, Ghd7 and PRR37 are both transcriptional suppressors (Weng et al. 2014; Liu et al. 2018). The effect of Ghd7 on heading date was the largest in the 3-gene segregating populations with fixed $H d 1$ genotypes, and the Ghd7 by PRR37 interaction was the strongest digenic interaction in these populations under NSD conditions (Table 2). Consequently, the enhanced genetic interaction between Ghd7 and PRR37 under SD conditions most likely attenuated the interaction of $G h d 7$ with other genes, and ultimately weakened the ability of Ghd7 and PRR37 or their complexes to inhibit the expression of downstream genes, Ehd 1 and $H d 3 a$, resulting in an early heading date. This hypothesis deserved to be further validated and improved by more genetic and molecular biology evidences.

\section{Conclusions}

Multi-order genetic interactions among Ghd7, Ghd8, PRR37 and $H d 1$ were observed in the 4-gene segregating population under both NLD and NSD conditions. These four genes jointly determined a large heading date variation and their homozygous combinations exhibited a continuous distribution under both conditions except two non-heading combinations under NLD conditions. Coupled with the correlation between heading date and SPP, the favorable combinations were suggested for local regions to maximize rice production. Furthermore, we revealed that PRR37 acted as a heading date suppressor under NLD conditions but it functioned alternatively under NSD conditions depending on the status of the other three genes, indicating different interactions among these four genes under different conditions. These findings revealed the importance of genetic interactions of these four genes in the photoperiod flowering pathways and contributed to a comprehensive insight into how these genes coordinate rice heading date under different day-length conditions. 


\section{Additional file}

Additional file 1: Figure S1. Development and genome composition of the rice populations. Figure S2. Genetic interaction analysis among Ghd7, Ghd8, PRR37 and Hd1 in the 4-gene segregating populations under NLD and NSD conditions. Figure S3. Genetic interaction analysis of Ghd7, Ghd8, PRR37 and Hd1 in the merged PRR37-segregating populations (NIL$\mathrm{F}_{4}$ ) under NLD and NSD conditions. Figure S4. PRR37 delays the heading date in the ghd7ghd8Hd1 background under both LD and SD conditions. Table S1. Characteristics of four heading date genes and linked markers. Table S2. The monthly average day length of growing seasons at Wuhan and Lingshui. Table S3. Haplotypes of 10 heading date genes in ZS97. Table S4. The genetic interactions in the 4-gene segregating populations under NLD and NSD conditions. Table S5. The genetic interactions among four genes on the basis of the merged PRR37-segragating populations $\left(\mathrm{NIL}-\mathrm{F}_{4}\right)$ under NLD and NSD conditions. Table S6. The heading date and spikelets per panicle of 16 homozygous 4-gene combinations under NLD and NSD conditions. Table S7. Primers used in this study. (DOCX $1337 \mathrm{~kb})$

\section{Abbreviations}

CCT: CO, CO-LIKE and TIMING OF CAB1; HAP: Heterotrimeric heme activator protein; LD: Long-day; NLD: Natural long-day; NSD: Natural short-day; QTLs: Quantitative trait loci; SD: Short-day; SPP: Spikelets per panicle; SSR: Simple sequence repeat

\section{Acknowledgments}

We would like to thank Mr. Jianbo Wang for his excellent work in the paddy field.

\section{Authors' contributions}

YX, HL and BZ planned and designed the research. BZ, HL and ZZ prepared the materials. BZ performed the experiments and data analysis, FQ and QL contributed to QTL genotyping. ZH contributed to data analysis. BZ and YX wrote and revised the manuscript. All authors read and approved the final manuscript.

\section{Funding}

This work was supported by the National Key Research and Development Program of China (2016YFD0100301), the National Natural Science Foundation of China (31701391, 31701054), and the Natural Science Foundation of Hubei Province (2015CFA006).

\section{Availability of data and materials}

The datasets supporting the conclusions of this article are included within the article and its additional files.

\section{Ethics approval and consent to participate}

Not applicable.

\section{Consent for publication}

Not applicable.

\section{Competing interests}

The authors declare that they have no competing interests.

Received: 11 January 2019 Accepted: 8 July 2019

Published online: 15 July 2019

\section{References}

Alexandrov N, Tai S, Wang W et al (2015) SNP-seek database of SNPs derived from 3000 rice genomes. Nucleic Acids Res 43:D1023-D1027

Andres F, Coupland G (2012) The genetic basis of flowering responses to seasonal cues. Nat Rev Genet 13:627-639

Bian XF, Liu X, Zhao ZG et al (2011) Heading date gene, dth3 controlled late flowering in O. Glaberrima Steud. By down-regulating Ehd1. Plant Cell Rep 30:2243-2254

Brambilla V, Fornara F (2013) Molecular control of flowering in response to day length in rice. J Integr Plant Biol 55:410-418
Doi K, Izawa T, Fuse T et al (2004) Ehd1, a B-type response regulator in rice, confers short-day promotion of flowering and controls FT-like gene expression independently of Hd1. Genes Dev 18:926-936

Fujino K, Yamanouchi U, Yano M (2013) Roles of the Hd5 gene controlling heading date for adaptation to the northern limits of rice cultivation. Theor Appl Genet 126:611-618

Gao H, Jin M, Zheng XM et al (2014) Days to heading 7, a major quantitative locus determining photoperiod sensitivity and regional adaptation in rice. Proc Natl Acad Sci U S A 111:16337-16342

Gao H, Zheng XM, Fei G et al (2013) Ehd4 encodes a novel and Oryza-genusspecific regulator of photoperiodic flowering in rice. PLoS Genet 9:e1003281

Goretti D, Martignago D, Landini M et al (2017) Transcriptional and posttranscriptional mechanisms limit heading date 1 ( $\mathrm{Hd} 1)$ function to adapt Rice to high latitudes. PLoS Genet 13:e1006530

Hayama R, Yokoi S, Tamaki S et al (2003) Adaptation of photoperiodic control pathways produces short-day flowering in rice. Nature 422:719-722

Hori K, Matsubara K, Yano M (2016) Genetic control of flowering time in rice: integration of Mendelian genetics and genomics. Theor Appl Genet 129: $2241-2252$

Hori K, Ogiso-Tanaka E, Matsubara K et al (2013) Hd16, a gene for casein kinase I, is involved in the control of rice flowering time by modulating the daylength response. Plant J 76:36-46

Kim SL, Lee S, Kim HJ et al (2007) OsMADS51 is a short-day flowering promoter that functions upstream of Ehd1, OsMADS14, and Hd3a. Plant Physiol 145: 1484-1494

Koo BH, Yoo SC, Park JW et al (2013) Natural variation in OsPRR37 regulates heading date and contributes to rice cultivation at a wide range of latitudes. Mol Plant 6:1877-1888

Kwon CT, Yoo SC, Koo BH et al (2014) Natural variation in early flowering1 contributes to early flowering in japonica rice under long days. Plant Cell Environ 37:101-112

Lee S, Kim J, Han JJ et al (2004) Functional analyses of the flowering time gene OsMADS50, the putative SUPPRESSOR OF OVEREXPRESSION OF CO $1 /$ AGAMOUS-LIKE 20 (SOC1/AGL20) ortholog in rice. Plant J 38:754-764

Lee YS, Jeong DH, Lee DY et al (2010) OsCOL4 is a constitutive flowering repressor upstream of Ehd1 and downstream of OsphyB. Plant J 63:18-30

Li F, Xie J, Zhu X et al (2018) Genetic basis underlying correlations among growth duration and yield traits revealed by GWAS in Rice (Oryza sativa L.). Front Plant Sci 9:650

Liu C, Qu X, Zhou Y et al (2018) OsPRR37 confers an expanded regulation of the diurnal rhythms of the transcriptome and photoperiodic flowering pathways in rice. Plant Cell Environ 41:630-645

Liu T, Liu H, Zhang H et al (2013) Validation and characterization of Ghd7.1, a major quantitative trait locus with pleiotropic effects on spikelets per panicle, plant height, and heading date in rice (Oryza sativa L.). J Integr Plant Biol 55:917-927

Matsubara K, Ogiso-Tanaka E, Hori K et al (2012) Natural variation in Hd17, a homolog of Arabidopsis ELF3 that is involved in Rice photoperiodic flowering. Plant Cell Physiol 53:709-716

Matsubara K, Yamanouchi U, Nonoue Y et al (2011) Ehd3, encoding a plan homeodomain finger-containing protein, is a critical promoter of rice flowering. Plant J 66:603-612

Matsubara K, Yamanouchi U, Wang ZX et al (2008) Ehd2, a rice ortholog of the maize INDETERMINATE1 gene, promotes flowering by up-regulating Ehd1. Plant Physiol 148:1425-1435

Murray MG, Thompson WF (1980) Rapid isolation of high molecular weight plant DNA. Nucleic Acids Res 8:4321-4325

Nemoto Y, Nonoue Y, Yano M et al (2016) Hd1, a CONSTANS ortholog in rice, functions as an Ehd 1 repressor through interaction with monocot-specific CCT-domain protein Ghd7. Plant J 86:221-233

Shibaya T, Hori K, Ogiso-Tanaka E et al (2016) Hd18, encoding histone Acetylase related to Arabidopsis FLOWERING LOCUS D, is involved in the control of flowering time in Rice. Plant Cell Physiol 57:1828-1838

Shrestha R, Gomez-Ariza J, Brambilla V et al (2014) Molecular control of seasonal flowering in rice, arabidopsis and temperate cereals. Ann Bot 114:1445-1458

Song JM, Lei Y, Shu CC et al (2018) Rice information GateWay: a comprehensive bioinformatics platform for Indica Rice genomes. Mol Plant 11:505-507

Song YH, Shim JS, Kinmonth-Schultz HA et al (2015) Photoperiodic flowering: time measurement mechanisms in leaves. Annu Rev Plant Biol 66:441-464

Statsoft I (1995) STATISTICA for windows (computer program manual). Statsoft Inc., Tulsa 
Takahashi Y, Shomura A, Sasaki T et al (2001) Hd6, a rice quantitative trait locus involved in photoperiod sensitivity, encodes the alpha subunit of protein kinase CK2. Proc Natl Acad Sci U S A 98:7922-7927

Takahashi Y, Teshima KM, Yokoi S et al (2009) Variations in Hd1 proteins, Hd3a promoters, and Ehd1 expression levels contribute to diversity of flowering time in cultivated rice. Proc Natl Acad Sci U S A 106:4555-4560

Tan J, Jin M, Wang J et al (2016) OsCOL10, a CONSTANS-like gene, functions as a flowering time repressor downstream of Ghd7 in Rice. Plant Cell Physiol 57:798-812

Thirumurugan T, Ito Y, Kubo T et al (2008) Identification, characterization and interaction of HAP family genes in rice. Mol Gen Genomics 279:279-289

Tsuji H, Taoka K, Shimamoto K (2011) Regulation of flowering in rice: two florigen genes, a complex gene network, and natural variation. Curr Opin Plant Biol 14:45-52

Wang W, Mauleon R, Hu Z et al (2018) Genomic variation in 3,010 diverse accessions of Asian cultivated rice. Nature 557:43-49

Wei X, Xu J, Guo H et al (2010) DTH8 suppresses flowering in rice, influencing plant height and yield potential simultaneously. Plant Physiol 153:1747-1758

Weng X, Wang L, Wang J et al (2014) Grain number, plant height, and heading date7 is a central regulator of growth, development, and stress response. Plant Physiol 164:735-747

Wenkel S, Turck F, Singer K et al (2006) CONSTANS and the CCAAT box binding complex share a functionally important domain and interact to regulate flowering of Arabidopsis. Plant Cell 18:2971-2984

Wu W, Zheng XM, Lu G et al (2013) Association of functional nucleotide polymorphisms at DTH2 with the northward expansion of rice cultivation in Asia. Proc Natl Acad Sci U S A 110:2775-2780

Xue W, Xing Y, Weng X et al (2008) Natural variation in Ghd7 is an important regulator of heading date and yield potential in rice. Nat Genet 40:761-767

Yamamoto E, Yonemaru J, Yamamoto T et al (2012) OGRO: the overview of functionally characterized genes in Rice online database. Rice (N Y) 5:26

Yan W, Liu H, Zhou X et al (2013) Natural variation in Ghd7.1 plays an important role in grain yield and adaptation in rice. Cell Res 23:969-971

Yan WH, Wang P, Chen HX et al (2011) A major QTL, Ghd8, plays pleiotropic roles in regulating grain productivity, plant height, and heading date in rice. Mol Plant 4:319-330

Yang W, Guo Z, Huang C et al (2014) Combining high-throughput phenotyping and genome-wide association studies to reveal natural genetic variation in rice. Nat Commun 5:5087

Yano K, Yamamoto E, Aya K et al (2016) Genome-wide association study using whole-genome sequencing rapidly identifies new genes influencing agronomic traits in rice. Nat Genet 48:927-934

Yano M, Katayose Y, Ashikari M et al (2000) Hd1, a major photoperiod sensitivity quantitative trait locus in rice, is closely related to the Arabidopsis flowering time gene CONSTANS. Plant Cell 12:2473-2484

Ye J, Niu X, Yang Y et al (2018) Divergent Hd1, Ghd7, and DTH7 alleles control heading date and yield potential of japonica Rice in Northeast China. Front Plant Sci 9:35

Yu H, Xie W, Li J et al (2014) A whole-genome SNP array (RICE6K) for genomic breeding in rice. Plant Biotechnol J 12:28-37

Zhang J, Zhou X, Yan W et al (2015a) Combinations of the Ghd7, Ghd8 and Hd1 genes largely define the ecogeographical adaptation and yield potential of cultivated rice. New Phytol 208:1056-1066

Zhang L, Li Q, Dong H et al (2015b) Three CCT domain-containing genes were identified to regulate heading date by candidate gene-based association mapping and transformation in rice. Sci Rep 5:7663

Zhang Z, Hu W, Shen G et al (2017) Alternative functions of Hd1 in repressing or promoting heading are determined by Ghd7 status under long-day conditions. Sci Rep 7:5388

Zhao J, Chen H, Ren D et al (2015) Genetic interactions between diverged alleles of early heading date 1 (Ehd1) and heading date $3 a(\mathrm{Hd} 3 \mathrm{a}) / \mathrm{RICE}$ FLOWERING LOCUS T1 (RFT1) control differential heading and contribute to regional adaptation in rice (Oryza sativa). New Phytol 208:936-948

Zhu S, Wang J, Cai M et al (2017) The OsHAPL1-DTH8-Hd1 complex functions as the transcription regulator to repress heading date in rice. J Exp Bot 68:553-568

\section{Publisher's Note}

Springer Nature remains neutral with regard to jurisdictional claims in published maps and institutional affiliations.

\section{Submit your manuscript to a SpringerOpen ${ }^{\circ}$ journal and benefit from:}

- Convenient online submission

- Rigorous peer review

- Open access: articles freely available online

- High visibility within the field

- Retaining the copyright to your article

Submit your next manuscript at $\boldsymbol{\nabla}$ springeropen.com 